\title{
Comparison of a New Insertion Element, IS1407, with Established Molecular Markers for the Characterization of Mycobacterium celatum
}

\author{
M. PICARDEAU, ${ }^{1}$ T. J. BULL,${ }^{2}$ G. PROD'HOM,${ }^{1}$ A. L. POZNIAK,${ }^{2}$ D. C. SHANSON,${ }^{3}$ AND V. VINCENT ${ }^{1 *}$ \\ Laboratoire de Référence des Mycobactéries, Institut Pasteur, 75724 Paris Cedex 15, France, ${ }^{1}$ and Kings College School \\ of Medicine and Dentistry, London SE 9RS, ${ }^{2}$ and Microbiology Department, Charing Cross and Westminster \\ Medical School, London W6 $8 R F,{ }^{3}$ United Kingdom
}

\begin{abstract}
Genomic analyses of 18 Mycobacterium celatum strains obtained from different patients in three countries (United States, United Kingdom, and France) were performed; the methods used in this study were restriction fragment length polymorphism (RFLP) analysis, pulsed-field gel electrophoresis (PFGE) analysis, and PCR restriction analysis (PRA) of the $h s p-65$ gene. A new insertion sequence, IS1407 (GenBank accession no. X97307), belonging to the IS256 family, was identified in $M$. celatum type 1 strains and was characterized by sequencing. When a probe for Mycobacterium xenopi IS1395-like sequences was used, the RFLP analysis of $M$. celatum type 1 strains revealed that they contained three or four copies of IS1407 in identical genomic positions, while this element was absent in all $M$. celatum type 2 strains. PFGE performed with three different endonucleases revealed a unique large restriction fragment (LRF) pattern for $M$. celatum type 1 strains, whereas the LRF patterns obtained for $M$. celatum type 2 strains were polymorphic. Moreover, PFGE of nondigested genomic DNA revealed extrachromosomal elements in $M$. celatum type 2 . The type strain of $M$. celatum type 3 could not be differentiated from $M$. celatum type 1 strains on the basis of the results of the RFLP analysis, the PFGE analysis, and the PRA of IS1407. In this study we confirmed that $M$. celatum types 1 and 2 represent distinct genomic clusters and that the molecular markers in $M$. celatum type 2 exhibit greater polymorphism than the molecular markers in $M$. celatum type 1 .
\end{abstract}

In 1993, Butler et al. described Mycobacterium celatum, a new slowly growing nonphotochromogenic species that has clinical importance (7). The first description of this species was based on the results of a study of clinical isolates recovered from bronchopulmonary specimens, as well as stool, cerebrospinal fluid, and blood specimens from human immunodeficiency virus-positive and -negative patients. The strains were obtained from diverse geographic areas in the United States. Two additional cases of infection by $M$. celatum have been reported in AIDS patients in Italy (20).

$M$. celatum strains have most of the phenotypic characteristics of Mycobacterium xenopi, Mycobacterium avium, and the recently described species Mycobacterium branderi (13). Gasliquid chromatography allows workers to differentiate $M$. celatum from both $M$. avium and $M$. xenopi on the basis of the presence of 2-eicosanol associated with tetracosanoic and hexacosanoic acids, which are the major mycolic acid cleavage products. However, this lipid pattern is also found in M. branderi. Genetic analysis of the 16S rRNA gene is the only method which has been described which can be used to reliably differentiate $M$. celatum from all other species. The $16 \mathrm{~S}$ rRNA sequences of $M$. celatum strains confirmed the close phylogenetic relationship of this organism to $M$. branderi and $M$. xenopi (level of similarity, 95\%) (7, 13). Moreover, sequence analysis has revealed that there is a 10-nucleotide difference between the sequences of $M$. celatum types 1 and 2 . Phenotypically, the two types could be differentiated only by multilocus enzyme electrophoresis, which resulted in two electrophoretic patterns based on the mobilities of 14 enzymes (7). Recently, 16S rRNA sequencing of additional $M$. celatum strains provided additional sequence information and revealed the existence of a

* Corresponding author. Phone: (33) (1) 456883 60. Fax: (33) (1) 406131 18. E-mail: vvincent@pasteur.fr. new sequence type that is distinct from but very similar to the type 1 and type 2 sequences; this new sequence type is designated type 3 (5).

A previous investigation of the host range of $M$. xenopi IS1395 revealed cross-hybridization with an unidentified repeated element in $M$. celatum type 1 (15). In order to investigate the molecular epidemiology of $M$. celatum further, we attempted to characterize this element and to evaluate the differences found in a group of $M$. celatum strains from diverse origins by using three molecular methods.

(This work is part of a doctoral thesis in microbiology presented by M.P.)

\section{MATERIALS AND METHODS}

Mycobacterial strains. The $M$. celatum reference strains and clinical isolate used in this study are listed in Table 1. Strains from the United States were kindly provided by W. R. Butler (Centers for Disease Control and Prevention, Atlanta, Ga.). M. celatum strains from the United Kingdom were provided by T. J. Bull (Kings College School of Dentistry and Medicine, London, United Kingdom) The $M$. branderi type strain (ATCC 51789) was a kind gift from M. L. Katila, Kuopio University Hospital, Kuopio, Finland. Other $M$. celatum isolates and two $M$. xenopi clinical isolates used as controls were from one of our laboratories (Laboratoire de Référence des Mycobactéries, Institut Pasteur, Paris, France). Identification by rRNA gene sequencing. The $16 \mathrm{~S}$ rRNA gene was amplified by using the PCR technique, and the sequence was determined as described previously with primers 244 and 259 (18). Sequencing reactions were performed with a model 373 stretch DNA analysis system (Applied Biosystems).

PCR restriction analysis (PRA). Approximately $1 \mu \mathrm{g}$ of mycobacteria was removed from Löwenstein-Jensen slants and suspended in $100 \mu$ l of TE (10 mM Tris, $1 \mathrm{mM}$ EDTA; pH 8.0) containing $1 \%$ Triton X-100 and incubated for 30 min at $100^{\circ} \mathrm{C}$. The resulting lysates were used as DNA sources without further purification. A two-step assay was then performed; this assay consisted of PCR amplification of a 439-bp fragment of the $h s p-65$ gene, followed by a restriction analysis performed with BstEII and HaellI as previously described (19). $M$. branderi and $M$. xenopi DNAs were used as controls.

Molecular cloning and library screening. Genomic DNA was prepared as previously described (15). A 3- $\mu \mathrm{g}$ portion of $M$. celatum type 1 DNA was digested with EcoRI and separated on a $0.8 \%$ (wt/vol) agarose gel. Restriction fragments that were approximately 2.5 to $5 \mathrm{~kb}$ long were recovered from the agarose gel by using a GeneClean II kit (Bio 101, Inc., La Jolla, Calif.) and were ligated into the 
TABLE 1. $M$. celatum strains used in this study

\begin{tabular}{llll}
\hline Strain & \multicolumn{1}{c}{ Designation } & \multicolumn{1}{c}{ Source $^{a}$} & Type \\
\hline 1 & CDC 911438 & Butler & 1 \\
2 & CDC 920541 & Butler & 1 \\
3 & CDC 900251 & Butler & 1 \\
4 & ATCC 51131 & Butler & $1^{b}$ \\
5 & CDC 892293 & Butler & $1^{b}$ \\
6 & CDC 890446 & Butler & 2 \\
7 & CDC 890694 & Butler & 2 \\
8 & CDC 920522 & Butler & $2^{b}$ \\
9 & ATCC 51130 & Butler & $2^{b}$ \\
10 & CDC 920605 & Butler & 2 \\
11 & CDC 890639 & Butler & 2 \\
12 & 960251 & Bull & $1^{b}$ \\
13 & 960252 & Bull & $1^{b}$ \\
14 & 960253 & Bull & $1^{b}$ \\
15 & NCTC 12882 & Bull & $3^{b}$ \\
16 & 941179 & Our laboratory & $1^{b}$ \\
17 & 940952 & Our laboratory & $1^{b}$ \\
18 & 951976 & Our laboratory & $1^{b}$ \\
\hline
\end{tabular}

"Butler. W. R. Butler, National Center for Infectious Diseases, Centers for Disease Control and Prevention, Atlanta, Ga.; Bull, T. J. Bull, Kings College School of Medicine and Dentistry, London, United Kingdom.

${ }^{b}$ The type was confirmed or established in this study by $16 \mathrm{~S}$ rRNA sequencing (see Materials and Methods).

EcoRI site of alkaline phosphatase-treated plasmid pBluescript II KS (Stratagene, La Jolla, Calif.). Ligation mixtures were transformed into Escherichia coli DH $5 \alpha$ by electroporation with a Gene Pulser unit (Bio-Rad Laboratories, Richmond, Calif.). Recombinant colonies were selected on Luria-Bertani solid medium ( $10 \mathrm{~g}$ of Bacto Tryptone [Difco] per liter, $5 \mathrm{~g}$ of yeast extract per liter, $10 \mathrm{~g}$ of $\mathrm{NaCl}$ per liter; $\mathrm{pH} 7.5$ ) supplemented with $100 \mu \mathrm{g}$ of ampicillin per $\mathrm{ml}, 2 \mathrm{mM}$ isopropyl- $\beta$-1)-thiogalactopyranoside, and $0.04 \%$-bromo-4-chloro-3-indolyl- $\beta$ D-galactopyranoside. Colonies were then transferred to a nylon filter $\left(\mathrm{N}^{+} \mathrm{Hy}\right.$ bond; Amersham International, Amersham, United Kingdom) by standard techniques (16).

A probe for IS1395-like sequences was prepared as described below and was hybridized to the filters overnight at $65^{\circ} \mathrm{C}$ in Rapid hybridization buffer (Amersham). This was followed by stringent washes at $65^{\circ} \mathrm{C}$; the preparations were washed twice $(10 \mathrm{~min}$ each) in $2 \times \mathrm{SSC}(1 \times \mathrm{SSC}$ is $0.15 \mathrm{M}$ sodium chloride plus $0.015 \mathrm{M}$ sodium citrate) containing $0.1 \%$ (wt/vol) sodium dodecyl sulfate (SDS), twice $(10 \mathrm{~min}$ each) in $1 \times \mathrm{SSC}-0.1 \%(\mathrm{wt} / \mathrm{vol}) \mathrm{SDS}$, and once for $10 \mathrm{~min}$ in $0.1 \times$ SSC-0.1\% (wt/vol) SDS.

Plasmid pMP02, which was used in later subcloning steps, was purified by using Qiagen minicolumns (Qiagen Midi kit; Qiagen, Inc., Hilden, Germany). The DNA insert was partially digested with Sau3A and was subcloned into the pBluescrip II $\mathrm{KS}^{-}$Bam HI site. Recombinant plasmids were extracted from $E$. coli by using Qiagen minicolumns for sequencing.

Sequencing and analysis of IS1407. The sequences of double-stranded plasmid DNAs were determined by the dideoxy chain termination method (17) by using a Taq DyeDeoxy terminator cycle sequencing kit (Applied Biosystems), a model 9600 GeneAmp PCR system (Perkin-Elmer), and a model 373 stretch DNA analysis system (Applied Biosystems). Similarities to the IS256 family were identified in databases by using the BLAST (1) and FASTA (14) algorithms.

Restriction fragment length polymorphism (RFLP). The IS1395 probe was amplified by PCR from an $M$. xenopi strain with primers XNA and XNB as previously lescribed (15). The 897-bp probe in IS1395 was purified from an agarose gel by the GeneClean procedure and was labeled with $\left[\alpha-{ }^{32} \mathrm{P}\right] \mathrm{dCTP}$ by using a Megaprime DNA labeling kit (Amersham).

A $2-\mu \mathrm{g}$ portion of mycobacterial DNA was digested with $10 \mathrm{U}$ of PvuII in a 30- $\mu$ l reaction mixture. DNA fragments were resolved by overnight electrophoresis on a $1 \%(\mathrm{wt} / \mathrm{vol})$ agarose gel at $1.5 \mathrm{~V} / \mathrm{cm}$ and were transferred to a nylon membrane ( $\mathrm{N}^{+}$Hybond; Amersham) (16). The membrane was hybridized overnight at $65^{\circ} \mathrm{C}$ with the probe in Rapid hybridization buffer (Amersham) and then washed as described above.

Pulsed-field gel electrophoresis (PFGE). $M$. celatum plugs were prepared as described previously (15). Since in the genus Mycobacterium the $\mathrm{G}+\mathrm{C}$ contents of the DNAs are very high, we used enzymes with AT-rich sites. Restriction endonucleases $A s e \mathrm{I}, \mathrm{XbaI}$, and $D r a \mathrm{I}$ were used to generate a few restriction fragments having lengths suitable for analysis. Large restriction fragments were separated in a $0.8 \%(\mathrm{wt} / \mathrm{vol})$ agarose gel by zero-integrated-field electrophoresis with the AutoBase system (Vysis, Les Ulis, France) for $65 \mathrm{~h}$ at room temperature with 8- to $200-\mathrm{kb}$ and 8- to 500-kb ROM cards.

Nucleotide sequence accession number. The GenBank accession number for the IS1407 DNA and amino acid sequences is X97307.

\section{A}

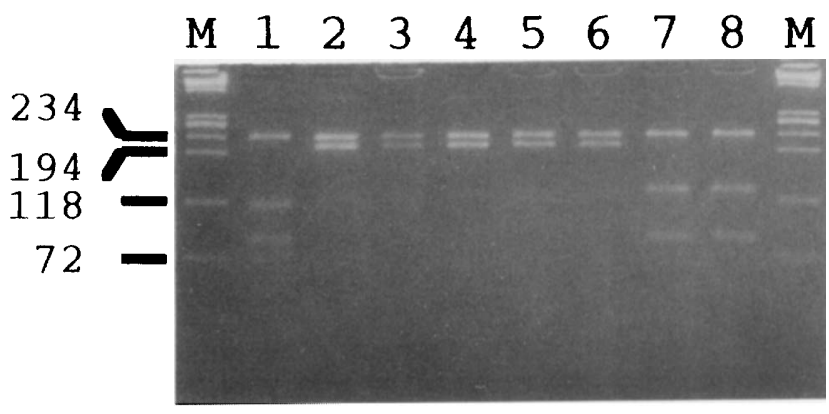

B

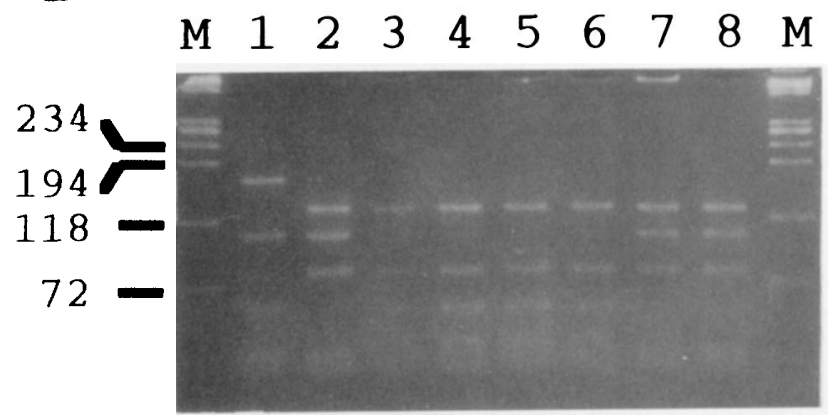

FIG. 1. (A) RFLP patterns generated by digestion with BstEII. (B) RFLP patterns generated by digestion with HaelII. Lanes M, molecular weight marker genome (molccular [in kilobases] are indicated on the left); lane 1, M.xenopi; lane $2, M$. branderi; lanes 3 to $5, M$. celatum type 1 strains 4,12 , and 13 , respectively; lane $6, M$. celatum type 3 strain 15 ; lanes 7 and $8, M$. celatum type 2 strains 9 and 11 , respectively.

\section{RESULTS}

PRA of the $\boldsymbol{h}$ sp-65 gene. The $M$. celatum type 1 strains and the type strain of $M$. celatum type 3 produced identical restriction patterns with the two enzymes used; the BstEII pattern had bands at 245 and $220 \mathrm{bp}$, and the HaeIII pattern had bands at 140 and $90 \mathrm{bp}$. The BstEII patterns of $M$. celatum type 2 strains contained 245-, 140-, and 80-bp fragments, and the HaeIII patterns of these strains contained 140- and 105-bp fragments. $M$. branderi and $M$. xenopi produced specific patterns. $M$. branderi produced the $M$. celatum type 1 and 3 pattern with BstEII and the M. celatum type 2 pattern with HaeIII, whereas $M$. xenopi produced dissimilar patterns with both enzymes (Fig. 1).

Description of insertion sequence IS1407. Screening of a partial library of the $M$. celatum type 1 genome with an IS1395 probe revealed a recombinant plasmid designated pMP002, which was purified and shown by restriction analysis to contain a 3.5-kb insert. A nucleotide sequence analysis of subclones of this plasmid showed that the element is $1,325 \mathrm{bp}$ long and contains at its extremities a 15-bp inverted repeat with one mismatch. The element contains a 1,245-bp open reading frame that encodes a putative 415-residue transposase preceded by a sequence which could serve as a ribosome binding site (data not shown). The putative transposase exhibits sequence homology to the Staphylococcus aureus IS256 family.

RFLP. Analysis of a Southern blot of PvuII-digested DNAs of 18 nonrelated strains, including $M$. celatum type 1 and 2 strains and the type strain of $M$. celatum type 3 , showed that 
1

$\begin{array}{llllllllll}2 & 3 & 4 & 5 & 6 & 7 & 8 & 9 & 10 & 11\end{array}$

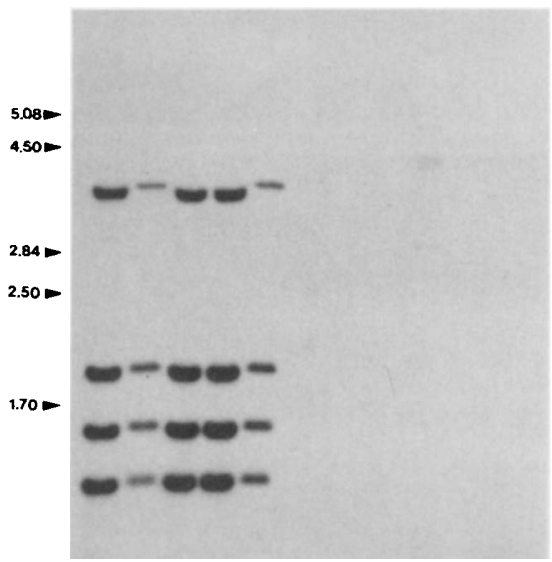

1213141516

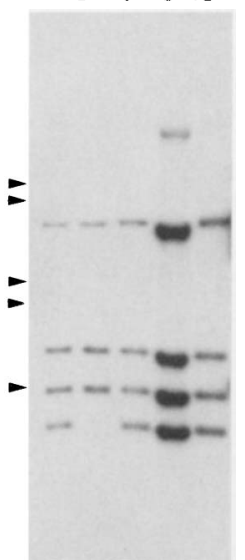

FIG. 2. Southern blot analysis of $P v u$ II-digested DNAs of $M$. celatum strains probed with IS1395. Lanes 1 to $5, M$. celatum type 1 strains 1 to 5 , respectively: lanes 6 to $11, M$. celatum type 2 strains 6 to 11 , respectively; lanes 12 to $15, M$. celatum type 1 strains 12 to 14 and 16 , respectively; lane $16, M$. celatum type 3 strains 15 . The molecular weight marker was bacteriophage $\lambda$ DNA restricted with PstI; the sizes of the fragments (in kilobases) are indicated on the left.

three or four copies of IS1407 were present in the $M$. celatum type 1 and 3 genomes. Nevertheless, the DNA fingerprints observed were not polymorphic. Identical patterns were obtained for most type 1 and type 3 strains; the only exceptions were two isolates that harbored one copy less. The element was not detected in any $M$. celatum type 2 strain (Fig. 2).

PFGE. $M$. celatum type 1,2 , and 3 genomes restricted with low-frequency cleavage enzymes DraI and AseI produced about 15 restriction fragments in the size range from 50 to 600 $\mathrm{kb}$. All of the patterns generated with $X b a$ I were complex (with more than 30 large restriction fragments [LRF]) and contained numerous fragments (Fig. 3).

The LRF patterns showed that the level genome conserva- tion in type 1 was high. The only difference found in the nonrelated type 1 strains was a $300-\mathrm{kb} X b a \mathrm{I}$ fragment (Fig. 3, lanes 2, 5, and 10). Moreover, the type 3 reference strain pattern was identical to the type 1 pattern. Unlike the type 1 and 3 patterns, the patterns for the $M$. celatum type 2 strains were polymorphic, which allowed differentiation of these organisms from each other. However, for each enzyme used, the strains tested shared about 10 LRF (Fig. 3).

The PFGE analysis of unrestricted DNA revealed extrachromosomal elements in $M$. celatum type 2 strains and the type 3 reference strain but not in $M$. celatum type 1 strains. Two of the $M$. celatum type 2 strains tested, strains 10 and 11 , harbored a $140-\mathrm{kb}$ plasmid, strain 8 harbored a $180-\mathrm{kb}$ plasmid, and strain 6 harbored a ca. 20 -kb element (Table 1 and Fig. 4). A 50-kb extrachromosomal element was detected in the $M$. celatum type 3 reference strain (Fig. 4). The PFGE migration of total DNA in the absence and presence of the intercalating agent ethidium bromide (data not shown) and the results obtained when different electrophoretic conditions were used suggested that these elements behaved like large linear plasmids similar to the $M$. xenopi extrachromosomal elements detected recently (Fig. 4) $(10,15)$.

\section{DISCUSSION}

The molecular epidemiology of $M$. celatum needs to be clarified, especially because of the association of this organism with disseminated infections in immunosuppressed patients. $M$. celatum was described for strains which had indistinguishable biochemical features but differed in their 16S rRNA sequences $(5,7)$. 16S rRNA sequencing revealed a 7-base difference between type 1 and type 3 and a 17-base difference between type 2 and type 3 (5). Previous studies have established that a cutoff value based on the number of different nucleotides in the 16S rRNA gene cannot be defined for delineating species $(11,24)$. For example, in the genus Mycobacterium, Mycobacterium szulgai and Mycobacterium malmoense differ by only a single nucleotide in the 1,384-nucleotide seg-
AseI

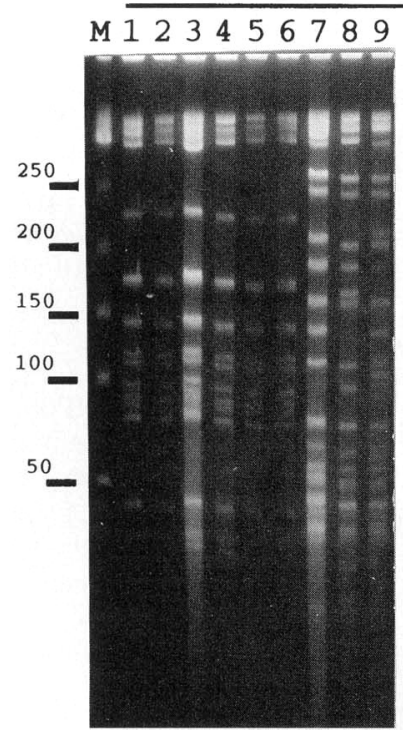

DraI

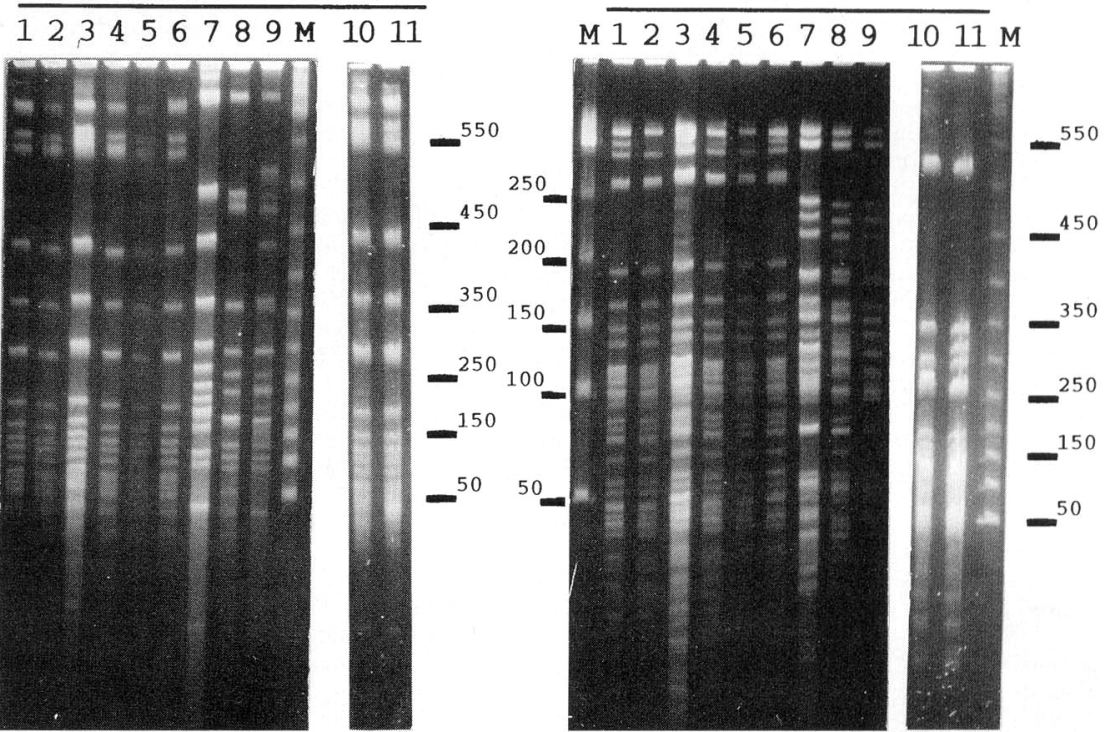

FIG. 3. PFGE separation of AseI-, DraI-, and XbaI-digested genomic DNAs from $M$. celatum strains. Lanes 1 to $6, M$. celatum type 1 strains $18,3,4$, and 12 to 14 , respectively; lanes 7 to $9, M$. celatum type 2 strains 9 to 11 , respectively; lane $10, M$. celatum type 1 strain 1 ; lane $11, M$. celatum type 3 strain 15 ; lanes $M$, molecular weight marker consisting of DNA concatemers of $50 \mathrm{~kb}$ of the bacteriophage $\lambda$ genome (the sizes [in kilobases] are indicated beside the gels). 


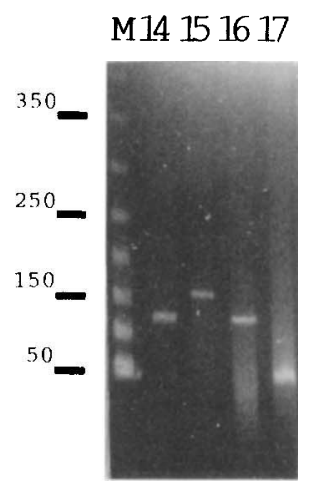

FIG. 4. PFGE of nondigested DNA: zero-integrated-field electrophoresis with the 8- to 200-kb ROM card (lanes 11 to 13) and with the 8- to 500-kb ROM card (lanes 1 to 10 and 14 to 17). Lanes 1 to $5, M$. celatum type 1 strains 1 to 5 , respectively; lanes 6 and $7, M$. celatum type 2 strains 7 and 9 , respectively; lanes 8 and 9 , $M$. celatum type 2 strains 10 and 11 , respectively; lanes 10 and $11, M$. xenopi; lane $12, M$. celatum type 2 strain 11 ; lane $13, M$. celatum type 2 strain 6; lane $14, M$. xenopi; lane $15, M$. celatum type 2 strain 8 ; lane $16, M$. celatum type 2 strain 10; lane 17, M. celatum type 3 strain 15; lanes M, molecular weight marker consisting of DNA concatemers of $50 \mathrm{~kb}$ of the bacteriophage $\lambda$ genome (the sizes [in kilobases] are indicated beside the gels).

ment examined, whereas some $M$. avium serotypes differ by one to seven nucleotides in the 782-nucleotide segment examined $(3,24)$. On the other hand, DNA-DNA hybridization studies and RFLP analyses established that $M$. malmoense and $M$. szulgai are distinct species and that the serotypes referred to above belong to a single species. Therefore, the 16S rRNA sequence variations observed in $M$. celatum types cannot be considered sufficient to assign the types to separate species, as Butler et al. (7) concluded previously.

We used three molecular methods to try to improve differentiation of the known $M$. celatum types and to distinguish strain variations within the types. A PRA of the $h s p-65$ gene showed excellent reproducibility, but could only be used to separate types 1 and 3 from type 2 . The patterns obtained by using BstEII and HaeIII confirmed the differentiation of types 1 and 2 described previously on the basis of results obtained with restriction enzymes Bst NI and XhoI (7). The number of restriction fragments indicate that these two $M$. celatum PRA types differ by a single restriction site for each enzyme. These results are indirect evidence that there is a high degree of conservation of the $h s p-65$ gene in these organisms. In addition to the conserved $h s p-65$ and $16 \mathrm{~S}$ rRNA genes, the superoxide dismutase (SOD) gene has also been used for taxonomic studies $(6,27)$. An analysis of partial SOD sequences did not reveal significant differences between $M$. celatum types 1 and 3 , yet there were significant differences between $M$. celatum types 3 and 2 (4).

It is possible, therefore, that the prevalence of $M$. celatum type 3 in our study was underestimated. It is interesting that all of the $M$. celatum strains identified outside the United States (i.e., the isolates identified in the United Kingdom, Italy, and France) were not type 2 strains $(5,19)$. $M$. celatum type 3 , as well as most of the $M$. celatum type 2 strains, harbored extrachromosomal elements ranging in size from about 20 to $180 \mathrm{~kb}$; none of these elements were detected in $M$. celatum type 1 strains (Fig. 4). However, the reliability of the presence of plasmids in $M$. celatum types must be confirmed by studying additional strains. These extrachromosomal elements are currently being investigated.

To determine strain differences within types, PFGE was used, and the results confirmed that $M$. celatum strains belong to two distinct genomic groups. The type 1 strains and the type strain of $M$. celatum type 3 belong to a compact cluster whose members produce a single PFGE pattern. Minor pattern differences were observed for some strains, which may have been due to DNA rearrangements. This homogeneity is in contrast to the great diversity described for other mycobacterial species, especially $M$. avium strains $(2,12,15,22,25,26)$. However, a lack of genomic polymorphism has been observed previously in the PFGE patterns of epidemiologically unrelated strains of Mycobacterium genavense, $M$. avium subsp. paratuberculosis, and $M$. avium subsp. silvaticum $(8,21)$. In contrast, PFGE provides valuable insight into the differentiation of $M$. celatum type 2 strains. Each of the strains tested produced a strainspecific pattern, but the patterns shared several LRF for each endonuclease tested. This finding is compatible with a high level of genetic relatedness among the strains.

In this study we identified a new insertion element, IS1407, that is found only in $M$. celatum types 1 and 3 . Insertion sequences have several characteristics that make them very useful for phylogenetic and epidemiological studies. A previous study showed that there was cross-hybridization of IS1395, an insertion sequence found recently in $M$. xenopi, with an element present in four copies in the genome of a $M$. celatum type 1 strain (15). In the present study the latter element was identified as IS1407, which belongs to the IS256 family. This finding expands the members of this family found in mycobacterial species, including IS1395 in M. xenopi, IS1081 in Mycobacterium bovis, IS 1245 and IS1311 in $M$. avium, and IS6120 in Mycobacterium smegmatis. The highest level of similarity between other sequences was the level of similarity with $M$. xenopi, a result which confirms the genetic relatedness of these mycobacterial species.

Unlike the other insertion elements belonging to the IS256 family described previously in mycobacteria, IS 1407 is not present in all types of its host species, as it could not be detected in $M$. celatum type 2 . Considering the high levels of homology between $M$. celatum types determined by $16 \mathrm{~S}$ rRNA and SOD gene sequence analyses, we suggest that the $M$. celatum types may have originated from a common ancestor. IS 1407 could have appeared in the $M$. celatum type 1 (and type 3 ) genomes by horizontal transfer after the divergence of types 1 and 2 or may have been lost by type 2 because of mutations that affected the function of the transposase, thereby causing a loss of the element.

The IS1407 RFLP patterns were identical for all $M$. celatum type 1 strains and the type strain of $M$. celatum type 3 (three or four copies at the same positions), although the isolates tested were from different countries (United States, United Kingdom, and France). Thus, IS1407 is not suitable as an epidemiological 
marker for strain differentiation. Moreover, an analysis of the flanking sequences of IS1407 revealed similarities with the flanking sequences of two related elements, $M$. bovis IS1081 and $M$. xenopi $\operatorname{IS} 1395(9,15)$ (data not shown). The absence of position polymorphism could be due to insertion of IS1407 at specific identical positions in the chromosome.

DNA-DNA hybridization, the reference technique used for species definition, could be used to conclusively determine the taxonomic hierarchy of the three $M$. celatum types. However, all of the results described above are consistent with the hypothesis that types 1 and 3 are closely related taxa and type 2 represents a separate species. A proposal for a new species name for type 2 is not presented here due to the recommendation of the Ad Hoc Committee on Reconciliation of Approaches to Bacterial Systematics (23) which requires defining phenotypic properties for species characterization.

In this study we demonstrated that $M$. celatum types 1 and 3 are very homogeneous. The results obtained for the type strain of $M$. celatum type 3 and the results obtained for the $M$. celatum type 1 strains were identical, as determined with the molecular markers used. Only the presence of an extrachromosomal element distinguished types 1 and 3. The molecular markers used in our study especially highlighted the close phylogenetic relationship of $M$. celatum with $M$. xenopi and $M$. branderi, which is consistent with the similar phenotypic properties of these species. The $h s p-65$ gene is highly conserved in $M$. celatum and $M$. branderi; in particular, the PRA profiles of $M$. branderi are either identical to the PRA profiles of $M$. celatum types 1 and 3 or identical to the PRA profiles of $M$. celatum type 2 , depending on the enzyme used. The three species harbor the related insertion sequences IS1407, IS1395, and IS1408 (GenBank accession no. U62766), respectively, all of which belong to the IS256 family and exhibit high levels of homology based on nucleotide and amino acid sequences.

\section{ACKNOWLEDGMENT}

M.P. is the recipient of a fellowship from Association Nationale de Recherches sur le SIDA.

\section{REFERENCES}

1. Altschul, S. F., W. Gish, W. Miller, E. W. Myers, and D. J. Lipman. 1990. Basic local alignment search tool. J. Mol. Biol. 215:403-410.

2. Arbeit, R. A., A. Slutsky, T. W. Barber, J. N. Maslow, S. Niemczyk, J. O. Falkinham, G. T. O'Connor, and C. F. von Reyn. 1993. Genetic diversity among strains of Mycobacterium avium causing monoclonal and polyclonal bacteremia in patients with AIDS. J. Infect. Dis. 167:1384-1390.

3. Boddinghaus, B., J. Wolters, W. Heikens, and E. C. Böttger. 1990. Phylogenetic analysis and identification of different serovars of Mycobacterium intracellulare at the molecular level. FEMS Microbiol. Lett. 70:197-204.

4. Bull, T. J., D. C. Shanson, and A. L. Pozniak. Unpublished data.

5. Bull, T. J., D. C. Shanson, L. C. Archard, M. D. Yates, M. E. Hamid, and D. E. Minnikin. 1995. A new group (type 3) of Mycobacterium celatum isolated from AIDS patients in the London aera. Int. J. Syst. Bacteriol. 45:861-862.

6. Bull, T. J., D. C. Shanson, and L. C. Archard. 1995. Rapid identification of mycobacteria from AIDS patients by capillary electrophoretic profiling of amplified SOD gene. JCP Clin. Mol. Pathol. 3:124-132.

7. Butler, W. R., S. P. O'Connor, M. A. Yakrus, R. W. Smithwick, B. B. Plikaytis, C. W. Moss, M. M. Floyd, C. L. Woodley, J. O. Kilburn, F. S. Vadney, and W. M. Gross. 1993. Mycobacterium celatum sp. nov. Int. J. Syst. Bacteriol. 43:539-548.

8. Cirillo, D., F. Torriani, P. Osegawa, S. Scotfield, and J. Fierer. 1996. Chro- mosomal DNA restriction patterns of clinical isolates of Mycobacterium genavense, abstr. I-169, p. 217. In Program and abstracts of the 36th Interscience Conference on Antimicrobial Agents and Chemotherapy. American Society for Microbiology, Washington, D.C.

9. Collins, D. M., and D. M. Stephens. 1991. Identification of insertion sequence, IS1081, in Mycobacterium bovis. FEMS Lett. 83:11-16

10. Ferdows, M. S., and A. G. Barbour. 1989. Megabase-sized linear DNA in the bacterium Borrelia burgdorferi, the Lyme disease agent. Proc. Natl. Acad. Sci. USA 86:5969-5973.

11. Fox, G. E., J. D. Wisotzkey, and P. Jurtshuk. 1992. How close is close: $16 \mathrm{~S}$ rRNA sequence identity may not be sufficient to guarantee species identity. Int. J. Syst. Bacteriol. 42:166-170.

12. Hector, J. S. R., Y. Pang, G. H. Mazurek, Y. Zhang, B. A. Brown, and R. J. Wallace. 1992. Large restriction fragment patterns of genomic Mycobacterium fortuitum DNA as strain-specific markers and their use in epidemiologic investigation of four nosocomial outbreaks. J. Clin. Microbiol. 30:1250-1255.

13. Koukila-Kähkölä, P., B. Springer, E. C. Böttger, L. Paulin, E. Jantzen, and M. L. Katila. 1995. Mycobacterium branderi sp. nov., a new potential human pathogen. Int. J. Syst. Bacteriol. 45:549-553.

14. Pearson, W. R., and D. J. Lipman. 1988. Improved tools for biological sequence comparison. Proc. Natl. Acad. Sci. USA 85:2444-2448.

15. Picardeau, M., A. Varnerot, J. Rauzier, B. Gicquel, and V. Vincent. 1996. Mycobacterium xenopi IS1395, a novel insertion sequence expanding the IS256 family. Microbiology 142:2453-2461.

16. Sambrook, J., E. F. Fritsch, and T. Maniatis. 1989. Molecular cloning: a laboratory manual, 2nd ed. Cold Spring Harbor Laboratory, Cold Spring Harbor, N.Y.

17. Sanger, F., S. Nicklen, and A. R. Coulson. 1977. DNA sequencing with chain-terminating inhibitors. Proc. Natl. Acad. Sci. USA 74:5463-5467.

18. Springer, B., L. Stockman, K. Teschner, G. D. Roberts, and E. Böttger. 1996 Two-laboratory collaborative study on identification of mycobacteria: molecular versus phenotypic methods. J. Clin. Microbiol. 34:296-303.

19. Telenti, A., F. Marchesi, M. Balz, F. Bally, E. C. Böttger, and T. Bodmer 1993. Rapid identification of mycobacteria to the species level by polymerase chain reaction and restriction enzyme analysis. J. Clin. Microbiol. 31:175178

20. Tortoli, E., C. Piersimoni, D. Bacosi, A. Bartoloni, F. Betti, L. Bono, C. Burrini, G. De Sio, C. Lacchini, A. Mantella, P. Giulo Orsi, V. Penati, M. Tullia Simonetti, and E. C. Böttger. 1995. Isolation of the newly described species Mycobacterium celatum from AIDS patients. J. Clin. Microbiol. 33: 137-140.

21. Vincent Lévy-Frébault, V., M. F. Thorel, A. Varnerot, and B. Gicquel. 1989 DNA polymorphism in Mycobacterium paratuberculosis, "wood pigeon mycobacteria," and related mycobacteria analyzed by field inversion gel electrophoresis. J. Clin. Microbiol. 27:2823-2826.

22. Wallace, R. J., Jr., Y. Zhang, B. A. Brown, V. Fraser, G. H. Mazurek, and S. Maloney. 1993. DNA large restriction fragment patterns of sporadic and epidemic nosocomial strains of Mycobacterium chelonae and Mycobacterium abscessus. J. Clin. Microbiol. 31:2697-2701.

23. Wayne, L. G., D. J. Brenner, R. R. Colwell, P. A. D. Grimont, O. Kandler, M. I. Krichevsky, L. H. Moore, W. E. C. Moore, R. G. E. Murray, E Stackebrandt, M. P. Starr, and H. G. Trüper. 1987. Report of the Ad Hoc Committee on Reconciliation of Approaches to Bacterial Systematics. Int. J. Syst. Bacteriol. 37:463-464.

24. Wayne, L. G., R. C. Good, E. C. Böttger, R. Butler, M. Dorsch, T. Ezaki, W. Gross, V. Jonas, J. Kilburn, P. Kirschner, M. I. Krichevsky, M. Ridell, T. M. Shinnick, B. Springer, E. Stackebrandt, I. Tarnok, Z. S. Tarnok, H. Tasaka, V. Vincent, N. G. Warren, C. A. Knott, and R. Johnson. 1996. Semantideand chemotaxonomic-based analyses of some problematic phenotypic clusters of slowly growing mycobacteria: a cooperative study of the International Working Group on Mycobacterial Taxonomy. Int. J. Syst. Bacteriol. 46:280. 297.

25. Yakrus, M. A., and W. L. Straus. 1994. DNA polymorphisms detected in Mycobacterium haemophilum by pulsed-field get electrophoresis. J. Clin. Microbiol. 32:1083-1084.

26. Zhang, Y., G. H. Mazurek, M. D. Cave, K. D. Eisenach, Y. Pang, D. T. Murphy, and R. J. Wallace, Jr. 1992. DNA polymorphisms in strains of Mycobacterium tuberculosis analyzed by pulsed-ficld gel electrophoresis: a tool for epidemiology. J. Clin. Microbiol. 30:1551-1556.

27. Zolg, J. W., and S. Philippi-Schulz. 1994. The superoxide dismutase gene, a target for detection and identification of mycobacteria by PCR. J. Clin. Microbiol. 32:2801-2812. 